\title{
Variación de la abundancia y diversidad de las aves migratorias en el humedal de Caucato, Pisco Perú
}

\author{
Variation in the abundance and diversity of migratory birds in the Caucato wetland, Pisco Peru \\ Juan Alberto Pisconte Vilca \\ juan.pisconte@ unica.edu.pe - Universidad Nacional San Luis Gonzaga, Ica \\ José Carlos Anchante Aparcana \\ j.anchante95@gmail.com - Universidad Nacional San Luis Gonzaga, Ica \\ Miguel Angel Aparcana Mendoza \\ maaparcana_m@ hotmail.com - Universidad Nacional San Luis Gonzaga, Ica \\ Joselyn Elizabeth Chipana Parvina \\ jchipanap.08@gmail.com - Universidad Nacional San Luis Gonzaga, Ica \\ Leidy Milady Ramos Alarcón \\ blga.leidyramosalarcon@gmail.com - Reserva Nacional Pampa Galeras
}

Recibido el 20/09/20 | Aceptado el 03/10/20

DOI: https://doi.org/10.47190/nric.v3i1.132

\section{Resumen}

En su ruta migratoria, numerosas aves llegan a los humedales Pisco; sin embargo, se conoce poco de ellas, así como del humedal de Caucato. Se estudió el ensamble, la variación de la abundancia y diversidad de la avifauna. Se realizaron conteos mensuales por hábitat empleando transectos de $100 \mathrm{~m}$ de largo y 20 metros de ancho en ambos lados. Para el ensamble de aves se usó la Clasificación de las especies de aves de América del Sur. La diversidad de Shannon-Wiener y la equitatividad de Pielou se calcularon con el software PAST 3.24; el análisis de componentes principales se usó para reducir la dimensionalidad de los registros de aves en los hábitats. Se registran 21 especies; las familias más representativas fueron Scolopacidae y Laridae. El hábitat con mayor abundancia y diversidad fue orilla de laguna (1335 individuos, $\mathrm{H}^{\prime}=2.26$ bits/ind. y $\mathrm{J}=0.81$ ), seguido de orilla de playa (837 individuos, $\mathrm{H}^{\prime}=2.18$ bits/ind. y $\mathrm{J}=0.81$ ) y espejo de agua (618 individuos, $\mathrm{H}^{\prime}=2.04$ bits/ind. y $\mathrm{J}=0.79$ ). Las variaciones en la abundancia y diversidad varían por la presencia de especies migratorias boreales o australes. Se concluye que el ensamble de la avifauna está dominado por los Scolopacidae y Laridae. La variación en la abundancia y diversidad mensual y por hábitats se relacionan con los ritmos migratorios de la avifauna y que, la orilla de laguna, orilla de playa y espejo de agua son los hábitats de mayor diversidad y abundancia.

Palabras clave: Ensamble, abundancia, diversidad, hábitats.

\begin{abstract}
On their migratory route, numerous birds arrive at the Pisco wetlands; however, little is known about them, as well as about the Caucato wetland. The assemblage, the variation of the abundance and diversity of the avifauna were studied. Monthly counts were made by habitat using transects $100 \mathrm{~m}$ long and $20 \mathrm{~m}$ wide on both sides. For the assemblage of birds the Classification of the species of birds of South America was used. Shannon-Wiener diversity and Pielou fairness were calculated with the PAST 3.24 software; principal component analysis was used to reduce the dimensionality of bird records in habitats. 21 species are registered; the most representative families were Scolopacidae and Laridae. The habitat with the highest abundance and diversity was the lagoon shore (1335 individuals, $H^{\prime}=2.26$ bits / ind. and $J=0.81$ ), followed by the beach shore ( 837 individuals, $H^{\prime}=2.18$ bits / ind. and $\mathrm{J}=0.81$ ) and water mirror (618 individuals, $\mathrm{H}^{\prime}=2.04 \mathrm{bits} / \mathrm{ind}$. and $\mathrm{J}=0.79$ ). Variations in abundance and diversity vary due to the presence of migratory boreal or southern species. It is concluded that the avifauna assemblage is dominated by the Scolopacidae and Laridae. The variation in monthly abundance and diversity and by habitats are related to the migratory rhythms of the birds and that the lagoon shore, beach shore and water mirror are the habitats with the greatest diversity and abundance.
\end{abstract}

Keywords: Assemblage, abundance, diversity, habitats.

\footnotetext{
Como citar: Pisconte-Vilca, J.A., Anchante-Aparcana, J.C., Aparcana Mendoza, M.A., Chipana-Parvina, J.E., \& Ramos-Alarcón, L.M. (2020). Variación de la abundancia y diversidad de las aves migratorias en el humedal de Caucato, Pisco Perú. NAWPARISUN - Revista de Investigación Científica, 3(1), 103-110.
} 


\section{Introducción}

Los humedales naturales existentes en la actualidad cubren sólo una fracción de su área original y han disminuido progresivamente durante siglos en la mayor parte del mundo (Convención Ramsar, 2018), siendo el cambio climático el principal factor del cambio ambiental global, y genera condiciones que modifican profundamente los atributos de vulnerabilidad natural y añadida (socioecológica) de los humedales (Franco Vidal, et al., 2013). Estos humedales son ecosistemas muy importantes que albergan una gran diversidad de flora y fauna, asimismo brindan múltiples servicios ecosistémicos; sin embargo, se encuentran entre los ecosistemas más amenazados del mundo (Clarkson et al. 2004), por el mal uso que se les da, causando degradación y pérdida del mismo (Secretaría de la Convención de Ramsar, 2013; Ruiz, 2014).

En la región desértica de la costa peruana, los humedales adquieren gran importancia al constituirse en verdaderos oasis para el paso de las aves migratorias, así como sinónimo de supervivencia de las comunidades de aves acuáticas residentes (Pulido, 2003); estos humedales costeros son uno de los ecosistemas más productivos; por sus características físicas y químicas, son ambientes ricos en flora y fauna y de gran valor ecológico dando sustento a altas concentraciones de aves (Keddy, 2000) tanto residentes como migratorias.

García-Olaechea (2018) citando a varios autores, señala que las aves migratorias usan temporalmente los humedales; asimismo menciona que la riqueza y abundancia de aves en los humedales es mayor en los meses de verano-otoño (diciembre-junio), con respecto a los meses de invierno-primavera (julionoviembre), debido a la llegada y partida de las especies de aves migratorias, respectivamente. También señala que entre las aves migratorias que arriban a los humedales costeros del Perú, se encuentran los chorlos y playeros de las familias Charadriidae, Haematopodidae, Recurvirostridae y Scolopacidae. En su viaje migratorio, estas aves llegan en primavera-verano aumentando la riqueza y abundancia de los ensambles de aves; asimismo, estos parámetros disminuyen hacia el otoño, coincidiendo con la época en que comienza el retorno migratorio hacia el hemisferio norte. Del mismo modo, aves playeras se dispersan ampliamente en sus áreas reproductivas, ocupando los parches de hábitats apropiados para la nidificación y cría; sin embargo, durante la migración y período no reproductivo se concentran en grandes números en determinados humedales costeros, aumentando su vulnerabilidad.

Pulido et al., (2020) menciona que las aves acuáticas forman parte importante de la biodiversidad y su tendencia de concentrarse en abundantes poblaciones, las hace vulnerables debido a que dependen de los humedales que son altamente productivos y les proporcionan un lugar para el descanso y alimentación; asimismo señala que, un número significativo de especies de aves acuáticas son migratorias neotropicales, neárticas, australes y andinas y utilizan los humedales durante el período de migración. Debido a la sensibilidad de las aves a los cambios ambientales, son utilizadas como indicadores biológicos en los diferentes tipos de hábitats. Por su posición en la cadena trófica, se ven afectadas por la pérdida de hábitat y por las diferentes condiciones físicas de los humedales, influyendo directamente en su abundancia y diversidad (Torres, 2006).

En diversos estudios de aves playeras en humedales, se encuentra variabilidad espacial y temporal en su distribución, así como variabilidad en la riqueza y abundancia en hábitats (Pulido et al., 2020); asimismo, la variación en la abundancia está asociado al tipo de hábitat, especialmente los hábitats limosos por el tipo de alimentación que presenta este grupo de aves (Torres et al., 2006).

El humedal de Caucato alberga una variada avifauna y es lugar de parada de las aves en su viaje migratorio hacia la parte más austral de Sudamérica; sin embargo, se conoce muy poco acerca los aspectos ecológicos de la avifauna asociada a este lugar. El propósito de la investigación fue determinar el ensamble de avifauna y conocer la variación de la abundancia y diversidad de las aves migratorias con la finalidad de brindar información que contribuya a su conservación. 


\section{Materiales y métodos}

\section{A) Área de Estudio}

El estudio se realizó en el humedal de Caucato, en la provincia de Pisco, Ica-Perú; ubicado en las coordenadas: $13^{\circ} 40^{\prime} 02^{\prime \prime}$ LS. y $76^{\circ} 12^{\prime} 47^{\prime \prime}$ LW. Este humedal limita con campos de cultivo, asimismo, el río Pisco desemboca a poca distancia del humedal y presenta una barrera de arena que lo separa del mar (Figura 1).

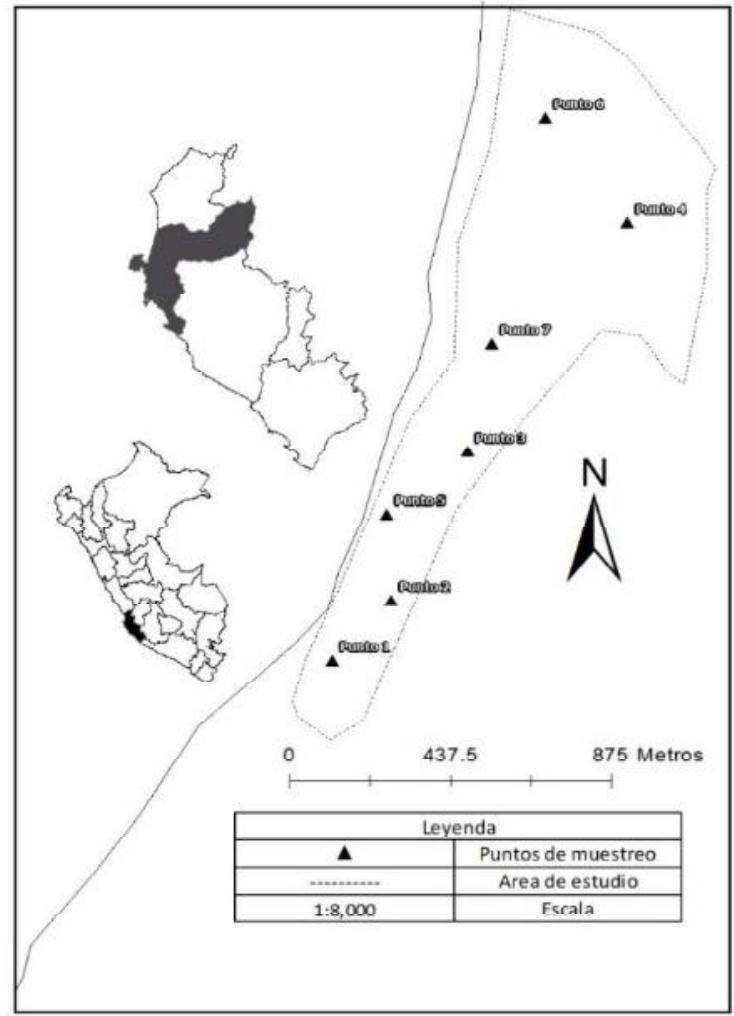

Figura 1. Ubicación de las zonas de estudio

B) Métodos.

\section{B.1) Recolección de datos}

Se identificaron 8 hábitats de acuerdo a la dominancia de la vegetación; asimismo, se anotó la vegetación asociada a dicho lugar (Tabla 1)

Tabla 1.

Vegetación asociada a cada hábitat

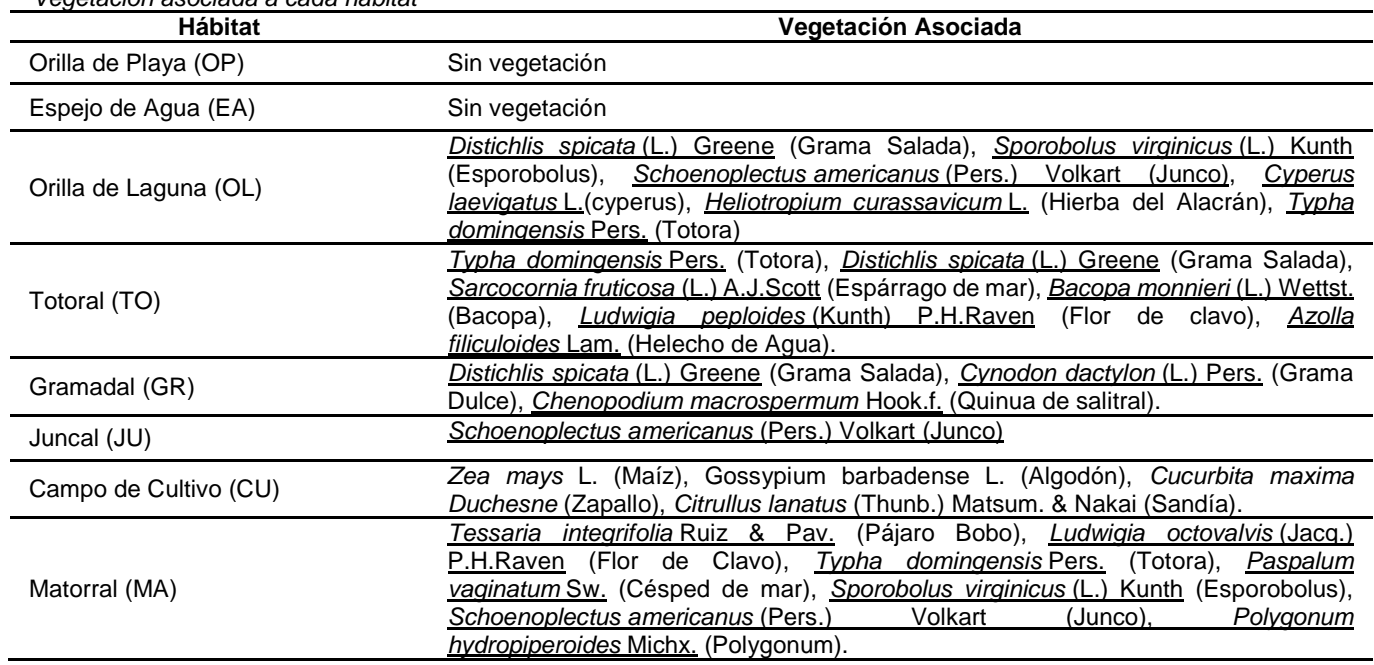


Se realizaron conteos de aves de enero a abril de 2017, mayo a agosto de 2018 y, setiembre a diciembre de 2019 . Se realizaron modificaciones a la propuesta de Bibby et al., (2000), contando mensualmente las aves en cada hábitat durante dos días consecutivos, empleando 2 transectos por estación de muestreo con franjas limitada a 20 metros en ambos lados y con una longitud de 100 metros.

La identificación de las aves se realizó mediante observaciones directas y las especies se determinaron siguiendo a Clements \& Shany (2001) y Schulenberg et al (2010).

\section{B.2. Análisis de datos}

La composición de especies se determinó a partir de la clasificación de las especies de aves de América del Sur (Remsen et. al 2020).

Se utilizó el software PAST 3.24 (Hammer et al., 2001) para calcular la diversidad de Shannon-Wiener (H') y la equitatividad de Pielou $(\mathrm{J})$; también se realizó un análisis de componentes principales para reducir la dimensionalidad de los registros de aves en los hábitats del humedal

\section{Resultados y Discusión}

\section{Ensamble de especies}

Se registran 21 especies pertenecientes a 5 familias (Tabla 2). De acuerdo a las categorías de la UICN, 2 especies se encuentran categorizadas como casi amenazadas (NT) y 19 en la categoría de preocupación menor (LC), motivo por el cual, el humedal de Caucato ha de considerarse como lugar importante para el refugio y alimentación de Ias aves migratorias.

El ensamble de la avifauna estuvo dominado por la familia Scolopacidae (11 especies) seguido de Laridae (6 especies). Las familias Charadriidae, Haematopodidae, Hirundinidae, Laridae y Scolopacidae han sido registradas en diversos humedales de la costa peruana: Pantanos de Villa (Pulido, 2018); Laguna El Paraíso (Quiñones y Hernández, 2017); Lagunas de Eten (Angulo et al., 2010); Laguna Cerro Negro (Pollack, 2017); Lagunas de Ite (Vizcarra, 2008); Lagunas de Ventanilla (Carazas, et al., 2015); sin embargo, no se han registrado la familia Pandionidae. En la investigación observamos a Pandion haliaetus sobrevolando el espejo de agua en actitud de forrajeo.

Tabla 2

Ubicación taxonómica de las especies registradas en el humedal de Caucato.

\begin{tabular}{|c|c|c|c|c|c|c|c|c|c|c|c|}
\hline Familia/Especies & UICN & OP & $\mathrm{EA}$ & $\mathrm{OL}$ & TO & GR & JU & $\mathrm{CU}$ & MA & Total & A.R \\
\hline $\begin{array}{l}\text { Charadriidae } \\
\text { Charadrius semipalmatus (Bonaparte, 1825) }\end{array}$ & LC & $\begin{array}{r}298 \\
15\end{array}$ & $\begin{array}{r}24 \\
7\end{array}$ & $\begin{array}{l}98 \\
81\end{array}$ & & & $\begin{array}{l}14 \\
14\end{array}$ & & & $\begin{array}{l}434 \\
117\end{array}$ & $\begin{array}{r}13.55 \\
3.65\end{array}$ \\
\hline Pluvialis squatarola (Linnaeus, 1758) & LC & 283 & 17 & 17 & & & & & & 317 & 9.89 \\
\hline Hirundinidae & & & & & 176 & 9 & 9 & 17 & 2 & 213 & 6.65 \\
\hline Hirundo rustica (Linnaeus, 1758) & LC & & & & 176 & 9 & 9 & 17 & 2 & 213 & 6.65 \\
\hline Laridae & & 357 & 401 & 454 & & & & & & 1212 & 37.83 \\
\hline Leucophaeus modestus (Tschudi, 1843) & LC & 52 & 97 & & & & & & & 149 & 4.65 \\
\hline Leucophaeus pipixcan (Wagler, 1831) & LC & 130 & 202 & 422 & & & & & & 754 & 23.53 \\
\hline Sterna hirundo (Linnaeus, 1758) & LC & 51 & & & & & & & & 51 & 1.59 \\
\hline Thalasseus maximus (Boddaert, 1783) & LC & 60 & 78 & 25 & & & & & & 163 & 5.09 \\
\hline Thalasseus elegans (Gambel, 1849) & NT & 46 & 24 & & & & & & & 70 & 2.18 \\
\hline Thalasseus sandvicensis (Latham, 1787) & LC & 18 & & 7 & & & & & & 25 & 0.78 \\
\hline Pandionidae & & & 4 & & & & & & & 4 & 0.12 \\
\hline Pandion haliaetus (Linnaeus, 1758) & LC & & 4 & & & & & & & 4 & 0.12 \\
\hline Scolopacidae & & 182 & 189 & 783 & 61 & 26 & 96 & 4 & & 1341 & 41.85 \\
\hline Actitis macularius (Linnaeus, 1766) & LC & 8 & 47 & 96 & 6 & 1 & 7 & & & 165 & 5.15 \\
\hline Arenaria interpres (Linnaeus, 1758) & LC & 44 & 1 & 66 & & & & & & 111 & 3.46 \\
\hline Calidris alba (Pallas, 1764) & LC & 18 & 19 & 140 & & 19 & 1 & 4 & & 201 & 6.27 \\
\hline Calidris mauri (Cabanis, 1857) & LC & 12 & & 80 & & & 11 & & & 103 & 3.21 \\
\hline Calidris melanotos (Vieillot, 1819) & LC & & & 14 & & & & & & 14 & 0.44 \\
\hline Calidris minutilla (Vieillot, 1819) & LC & & & 91 & 26 & 4 & 43 & & & 164 & 5.12 \\
\hline Calidris pusilla (Linnaeus, 1766) & NT & 28 & & 155 & 27 & 2 & 10 & & & 222 & 6.93 \\
\hline Numenius phaeopus (Linnaeus, 1758) & $\mathrm{LC}$ & 70 & & 12 & & & 3 & & & 85 & 2.65 \\
\hline Phalaropus tricolor (Vieillot, 1819) & LC & 2 & 79 & 2 & & & & & & 83 & 2.59 \\
\hline Tringa flavipes (Gmelin, 1789) & LC & & 23 & 78 & 2 & & 17 & & & 120 & 3.75 \\
\hline Tringa melanoleuca (Gmelin, 1789) & LC & & 20 & 49 & & & 4 & & & 73 & 2.28 \\
\hline $\mathrm{N}^{\circ}$ de Especies & & 15 & 13 & 16 & 5 & 5 & 10 & 2 & 1 & & \\
\hline $\mathrm{N}^{\circ}$ de Individuos & & 837 & 618 & 1,335 & 237 & 35 & 119 & 21 & 2 & 3,204 & \\
\hline Shannon - Wiener $\left(\mathrm{H}^{\prime}\right)$ & & 2.18 & 2.04 & 2.26 & 0.84 & 1.19 & 1.94 & 0.49 & 0.00 & & \\
\hline Equitatividad (J) & & 0.81 & 0.79 & 0.81 & 0.52 & 0.74 & 0.84 & 0.70 & 0.00 & & \\
\hline
\end{tabular}

$(\mathrm{OL})$, totoral (TO), gramadal (GR), juncal (JU), campo de cultico (CU), matorral (MA). Abundancia relativa: A.R. 


\section{Abundancia}

Durante el periodo de estudio, se registró 3,204 individuos (Tabla 2); la mayor abundancia fue registrada en la orilla de laguna (1,335 individuos), seguido orilla de playa (837 individuos) y del espacio aéreo (618 individuos). Durante su permanencia en el humedal las aves fueron observadas en actitud de forrajeo o descansando en el espejo de agua, siendo el hábitat con mayor número de especies e individuos. Le siguen en abundancia la orilla de playa, hábitat que presenta un amplio espacio para el descanso y alimentación de las aves migratorias siendo ocupado principalmente por Leucophaeus pipixcan. Por otro lado, se observaron disturbios que alteraron la presencia de las aves (ruido de unidades móviles, presencia humana y animales domésticos), ocasionando que estuvieran en alerta, se desplacen dentro del mismo hábitat o realicen vuelos dentro del mismo humedal, logrando registrar en el espacio aéreo un significativo número de especies e individuos.

Las familias Scolopacidae y Laridae presentan las mayores abundancias (1,341 y 1,212 individuos respectivamente) y las especies con las poblaciones más numerosas fueron: Leucophaeus pipixcan (754 individuos) y Pluvialis squatarola (317 individuos).

El análisis de componentes principales (Figura 2) muestra que los hábitats matorral, campo de cultivo, gramadal totoral y juncal forman un grupo con una baja abundancia de individuos, mientras que el hábitat orilla de laguna, orilla de playa y espejo de agua son independientes y contienen la mayor abundancia.

La abundancia relativa, como un componente de la biodiversidad, se refiere a que tan común o rara es una especie en comparación con otras especies en una comunidad biológica o una ubicación definida; en este sentido, Pandion haliaetus, Calidris melanotos y Thalasseus sandvicensis son las especies de mayor rareza por la menor abundancia relativa registrada durante el periodo de estudio.

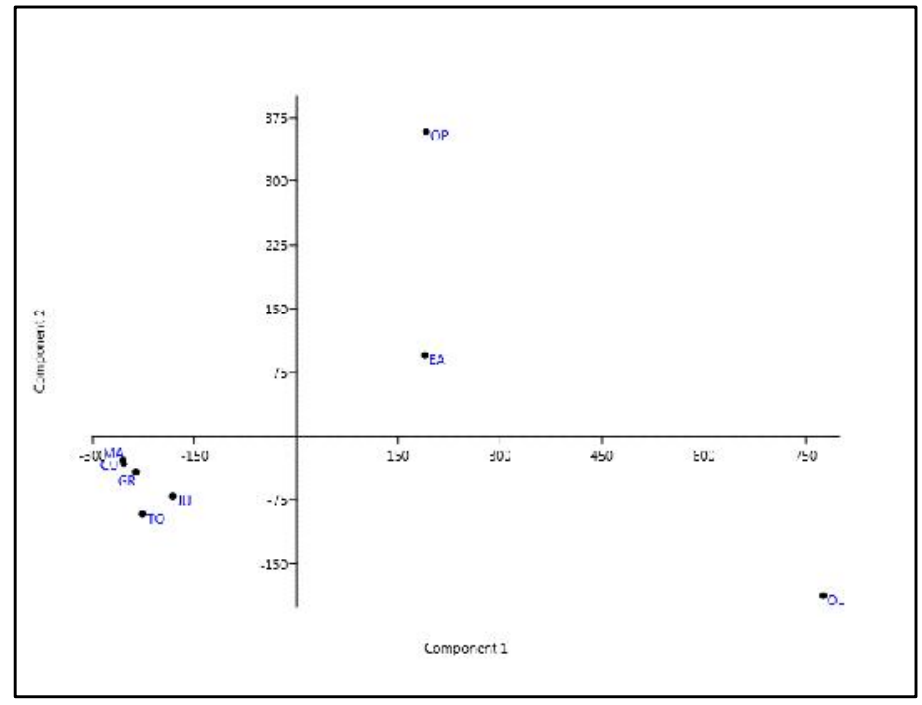

Figura 2. Análisis de componentes principales entre la abundancia de individuos por hábitat.

La tabla 3 y figura 3 muestran la variación de la abundancia de las aves migratorias; apreciándose tres etapas: 1) de enero a mayo el número de individuos disminuye progresivamente coincidiendo este hecho con el aumento del caudal del río Pisco, inundando el lugar y dejando menor espacio para la permanencia de las aves; 2) de junio a octubre se aprecia un ligero incremento; al respecto, Torres et al., (2006) citando a otros autores, mencionan que la presencia de individuos juveniles en un lugar es porque probablemente no llegan a migrar debido a que no se reproducen durante su primer año de vida y señalan que, en Calidris alba los juveniles no acumulan suficiente grasa para generar energía por lo que no podrían migrar y permanecen en los mismos lugares. En nuestro caso, observamos la presencia de individuos de Arenaria interpres y Tringa melanoleuca con plumaje reproductivo, lo que confirmaría lo señalado anteriormente. Estos resultados sugieren que las poblaciones de las especies migratorias encuentran en el humedal de Caucato un sitio de parada en su ruta migratoria. Durante el estudio (Tabla 3) también se registró la presencia de Leucophaeus modestus, una especie que se desplaza de la zona más austral de Sudamérica hasta las costas de Perú, influyendo en el crecimiento de la curva poblacional; 3) entre octubre y diciembre se incrementa el número de individuos, coincidiendo con la llegada de los primeros migratorios boreales a esta parte del continente sudamericano.

Es importante destacar que los datos obtenidos en el humedal de Caucato son los primeros registros y podrían ser utilizados para elaborar planes de manejo y conservación. Esta pequeña área, pese a estar relativamente perturbada, contribuye notablemente con los niveles de biodiversidad local; asimismo, la variedad de hábitats, disponibilidad de alimento y refugio ante depredadores, serían factores que influyen positivamente en la diversidad de aves en este ecosistema. 
Tabla 3

Conteo mensual de las especies registradas en el humedal de Caucato

\begin{tabular}{|c|c|c|c|c|c|c|c|c|c|c|c|c|}
\hline ESPECIES & ENE & FEB & MAR & ABR & MAY & JUN & JUL & AGO & SET & OCT & NOV & DIC \\
\hline Actitis macularius & 17 & 41 & 9 & & & & & 4 & 18 & 15 & 17 & 44 \\
\hline Arenaria interpres & & 19 & 1 & 29 & 4 & 1 & 3 & 3 & 14 & 15 & 3 & 19 \\
\hline Calidris alba & 1 & 15 & 2 & 14 & 9 & 18 & 22 & 18 & 13 & 9 & & 80 \\
\hline Calidris mauri & 15 & 24 & 22 & & & & & & 9 & 5 & 9 & 19 \\
\hline Calidris melanotos & & & & & & & & & 2 & 4 & 6 & 2 \\
\hline Calidris minutilla & 7 & 2 & 6 & & & 12 & 22 & 11 & 19 & 21 & 35 & 29 \\
\hline Calidris pusilla & 38 & 22 & 35 & & & & & 29 & 3 & 26 & 26 & 43 \\
\hline Charadrius semipalmatus & 2 & 11 & 2 & 6 & 2 & 21 & 5 & 5 & 1 & 19 & 22 & 21 \\
\hline Hirundo rustica & 18 & 17 & 22 & 18 & & & & & 2 & 19 & 32 & 85 \\
\hline Leucophaeus modestus & 11 & & 8 & & & 19 & 23 & 64 & 13 & & 7 & 4 \\
\hline Leucophaeus pipixcan & 178 & 104 & 24 & & & & & 2 & 39 & 57 & 149 & 201 \\
\hline Numenius phaeopus & 5 & 15 & 10 & & & & 2 & 6 & 15 & 5 & 6 & 21 \\
\hline Pandion haliaetus & & & & & & & & & 1 & 1 & 1 & 1 \\
\hline Phalaropus tricolor & 8 & 16 & 10 & & & & & 2 & 12 & 16 & 5 & 14 \\
\hline Pluvialis squatarola & 88 & 13 & 74 & & & & 16 & & 1 & 2 & & 123 \\
\hline Sterna hirundo & 8 & 28 & & & & & & & & & 15 & \\
\hline Thalasseus elegans & 22 & 9 & 25 & & & & 4 & & 27 & 8 & 51 & 17 \\
\hline & 2 & 10 & 7 & 2 & & & & 14 & & 6 & 4 & 25 \\
\hline Thalasseus sandvicensis & 5 & 4 & & & & & & & & 1 & 6 & 9 \\
\hline Tringa flavipes & 11 & 12 & 19 & 12 & & 3 & 14 & 4 & 8 & 6 & 13 & 18 \\
\hline Tringa melanoleuca & & 3 & 6 & 5 & & 2 & 17 & 10 & 17 & 3 & 5 & 5 \\
\hline $\mathrm{N}^{\circ}$ de especies & 17 & 18 & 17 & 7 & 3 & 7 & 10 & 13 & 18 & 19 & 19 & 20 \\
\hline & 436 & 365 & 282 & 86 & 15 & 76 & 128 & 172 & 214 & 238 & 412 & 780 \\
\hline Shannon - Wiener $\left(\mathrm{H}^{\prime}\right)$ & 1.99 & 2.49 & 2.41 & 1.70 & 0.93 & 1.62 & 2.07 & 2.02 & 2.54 & 2.53 & 2.27 & 2.42 \\
\hline Equitatividad ( J ) & 0.70 & 0.86 & 0.85 & 0.88 & 0.84 & 0.83 & 0.90 & 0.79 & 0.88 & 0.86 & 0.77 & 0.81 \\
\hline
\end{tabular}

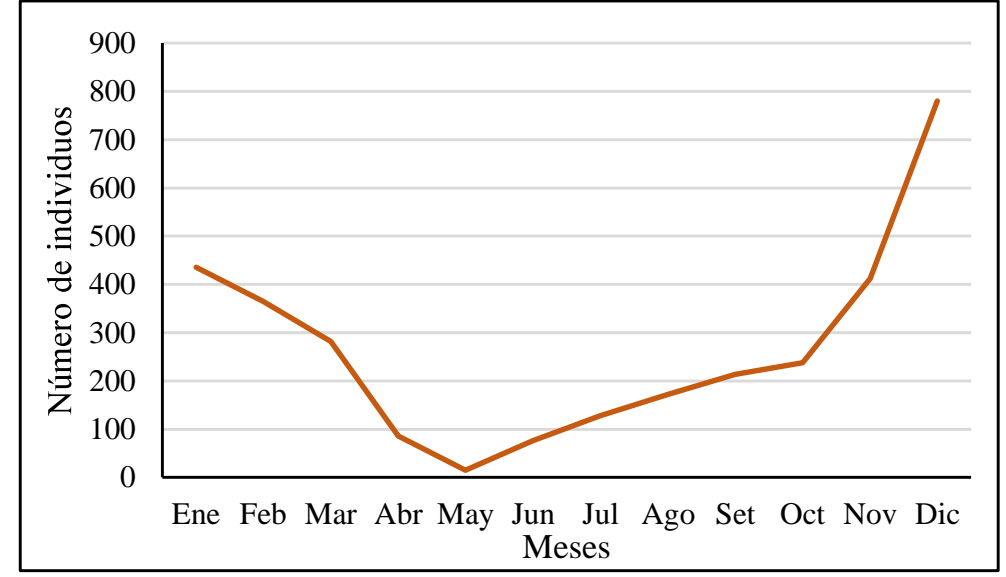

Figura 3. Variación mensual de la abundancia de aves migratorias en el humedal de Caucato.

\section{Diversidad}

En estudios realizados por lannacone et al (2010) en Pantanos de Villa entre los años 2004 y 2007, muestran índices de Shannon - Wiener con ligeras variaciones respecto a los índices registrados en el presente estudios (Tablas 2 y 3), donde se aprecia que la mayor diversidad y equitatividad corresponde a la orilla de laguna $\left(\mathrm{H}^{\prime}=2.26 \mathrm{bits} / \mathrm{ind}\right.$. y $\mathrm{J}=$ $0.81)$, seguida de la orilla de playa $\left(H^{\prime}=2.18\right.$ bits/ind. y $J=0.81$ y el espejo de agua $\left(H^{\prime}=2.04\right.$ bits/ind. y $\left.J=0.79\right)$. En la orilla de la laguna observamos la presencia de aves limícolas (Charadriidae y Scolopacidae) forrajeando; la orilla de playa es usada para el descanso de los Laridae, mientras que a los Charadriidae y Scolopacidae se les observó en actitud de forrajeo. En el espejo de agua las aves fueron observadas en descanso o alimentándose; asimismo, se registró a Pandion haliaetus sobrevolando el humedal en busca de presas. Al respecto, González et al. (2011) menciona que los humedales estuarinos son sistemas altamente dinámicos debido a la influencia de las mareas, y a la confluencia en zonas ecotonales entre el agua de mar y el agua proveniente de los ríos; estas características los convierten en hábitats clave de un importante número de especies de aves acuáticas. Asimismo, Pérez et al (2018) señalan que muchas especies migratorias realizan desplazamientos oportunistas en búsqueda de humedales con mínimos de supervivencia y que los cambios en la disponibilidad de áreas de al imentación y acceso a las presas, determinan la existencia de desplazamientos cortos a nivel local, relacionados con la búsqueda de sitios alternativos de forrajeo y áreas de dormidero. 
La Tabla 3 y la figura 4 muestran la variación mensual de los índices de diversidad, apreciándose el mismo comportamiento poblacional que el de la abundancia. Por otro lado, los análisis de diversidad y equidad del humedal de Caucato sugieren que este sistema mantiene poblaciones de aves que son dominantes en términos de su abundancia. Por otro lado, González et al. (2011) menciona que la alta representatividad numérica de individuos pertenecientes a unas familias de aves, así como la rareza de individuos de otras familias evidencia la baja equidad de este sistema, quizá en parte, a que las especies de estas familias usan el humedal de manera temporal (migración en época no reproductiva). Para nuestro caso, las familias Scolopacidae y Laridae presentan la mayor abundancia y la rareza de individuos se encuentra en las familias Hirundinidae y Pandionidae evidenciando una baja equidad de este sistema.

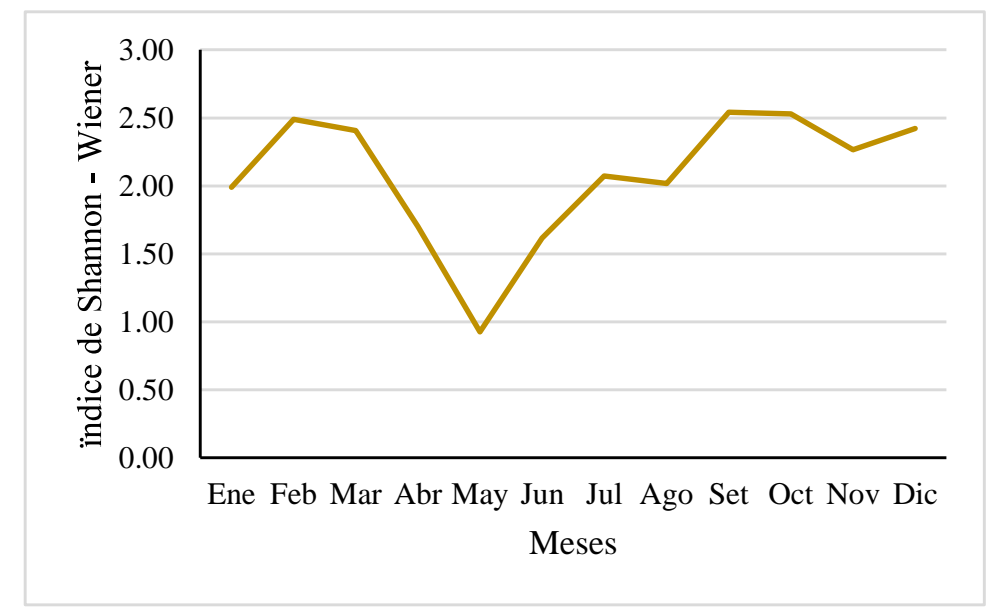

Figura 4. Variación mensual de los índices de diversidad.

\section{Conclusiones}

Se concluye que el ensamble de la avifauna está dominado por las familias Scolopacidae y Laridae. La variación en la abundancia y diversidad mensual y por hábitats guardan relación con los ritmos migratorios de la avifauna boreal y austral; asimismo, la orilla de laguna, orilla de playa y espejo de agua son los hábitats de mayor diversidad y abundancia. 


\section{Referencias bibliográficas}

Angulo, F., Schulenberg, T., \& Puse, E. (2010). Las aves de los humedales de Eten, Lambayeque, Perú. Ecología Aplicada. 9(2): 71-81.

Recuperado en:http://www.scielo.org.pe/scielo.php?script =sci_arttext\&pid=S1726 22162010000200002

Bibby, C J, Burgess, ND, Hill, D A y Mustoe, S. (2000). Técnicas de censo de aves. Elsevier.

Carazas, N., Camargo, L., Gil, F., Zárate, R. R., Liviac, R., Zárate, R. R., \& Jessie, M. (2015). Nuevos Registros De Fauna En El Área De Conservación Regional (ACR) Humedales De Ventanilla: Actualización. Científica, 12(1), 42-60.

Clarkson, B. R.; B. Sorrell; P. Reeves; P. Champion; T. Patridge \& B. D. Clarkson. (2004). Handbook for Monitoring Wetland Conditions. Ministry for the Environment Sustainable Management Fund Project (5105).

Clements J. \& Shany N. (2001). A Field Guide to the Birds of Peru. Temecula: Ibis Publishing Company.

Convención de Ramsar sobre los Humedales. (2018). Perspectiva Mundial sobre los humedales: Estado de los humedales del mundo y de los servicios que prestan a las personas 2018 . Informe Secretaria de la Convención de Ramsar. Gland (Suiza).

Franco Vidal, L., Delgado, J., \& Andrade, G. I. (2013). Factores de la vulnerabilidad de los humedales altoandinos de Colombia al cambio climático global. Cuadernos de Geografía: Revista Colombiana de Geografía, 22(2), 69-85. https://doi.org/10.15446/rcdg.v22n2.37018

García-Olaechea, A., Chávez-Villavicencio, C., \& Tabilo-Valdivieso, E. (2018). Do influence nearctic migratory birds in the seasonal pattern of coastal wetlands birds? Revista Peruana de Biología, 25(2), 117-122. https://doi.org/10.15381/rpb.v25i2.13281

González, A. L., Vukasovic, M. A., \& Estades, C. F. (2011). Variación temporal en la abundancia y diversidad de aves en el humedal del río Itata, región del Bío-Bío, Chile. Gayana, 75(2), https://doi.org/10.4067/S071765382011000200006

Hammer O., Harper, D.A.T. \& Rayan P.D. (2001). Past: paleontological statistics software package for education and data analysis. Paleontología electronica

lannacone J., Atasi, M., Bocanegra, T., Camacho, M., Montes, A., Santos, S., Alayo, M., \& Agustino, E. (2010). Diversidad de aves en el humedal Pantanos de Villa, Lima, Perú: periodo 20042007. Biota neotropical. Vol $10 \mathrm{~N}^{\circ} 2$.

Keddy, P.A. (2000). Wetland ecology: principles and conservation. Cambridge University Press. Cambrigde, UK.

Pérez A., De la Cruz M. Varela R., Puente M., Pérez E. (2018). Caracterización Del Ensamblaje De Aves Acuáticas Asociadas A Lagunas Litorales Del Sur De La Península De
Guanahacabibes, Cuba. Poeyana 507 julio diciembre 2018. 1-7.

Pollack Velásquez, L. E. (2017). Ensamble de aves del humedal de Cerro Negro, Puerto Morín, La Libertad. Arnaldoa, 24(2), 645-656. https://doi.org/10.22497/arnaldoa.242.24215

Pulido V. (2003). Influencia de la pérdida de hábitats en la conservación de las aves de los Pantanos de Villa. Tesis para optar el grado Académico de Doctor en Ciencias Biológicas. UNMSM.

Pulido, V. (2018). One hundred and fifty years of keep records from Pantanos de Villa birds' [Ciento quince años de registros de aves en Pantanos de Villa]. Revista Peruana de Biología, 25(3), 291-306. https://doi.org/10.15381/rpb.v25i3.15212

Pulido V, Salinas L, del Pino J, Arana C. (2020). Preferencia de hábitats y estacionalidad de las especies de aves de los Pantanos de Villa en Lima, Perú. Revista peruana de biología 27(3): 349 - 360 (agosto 2020). doi: http:// dx.doi.org/10.15381/rpb.v27i3.18681

Quiñones, A., \& Hernández, F. (2017). Uso de hábitat y estado de conservación de las aves en el humedal El Paraíso, Lima, Perú. Revista peruana de biología 24(2): 175 - 186 Recuperado en http://dx.doi.org/10.15381/rpb.v24i2.13494.

Remsen, JV, Jr., JI Areta, E. Bonaccorso, S. Claramunt, A. Jaramillo, JF Pacheco, C. Ribas, MB Robbins, FG Stiles, DF Stotz y KJ Zimmer. Versión 8 de junio de 2020. Una clasificación de las especies de aves de América del Sur. Sociedad Ornitológica Estadounidense.

http://www.museum.Isu.edu/ Remsen/SAC CBaseline.htm.

Ruiz, D.C. (2014). Análisis histórico y prospectiva del humedal Tierra Blanca. Perspectiva Geográfica, 19(1), 125-144.

Schulenberg, TS, Stotz, DF, Lane, DF, O'Neill, JP y Parker III, TA. (2010). Birds of Perú: edición revisada y actualizada. Prensa de la Universidad de Princeton.

Secretaría de la Convención de Ramsar. (2013). Manual de la convención de Ramsar: guía a la convención sobre los humedales (Ramsar, Irán, 1971). Gland: Secretaría de la convención de Ramsar. 120 p.

Torres M. (2006). "Dinámica temporal de la comunidad de aves (Haematopodidae, Charadriidae, Scolopacidae, Recurvirostridae y Phalaropodidae) en la Zona Reservada de los Pantanos de Villa durante el año 2005" Tesis para optar el título de Bióloga. Universidad Nacional Agraria - La Molina.

Torres, M.; Quinteros, Z. \& Takano, F. (2006). Variación temporal de la abundancia y diversidad de aves limícolas en el Refugio de Vida Silvestre Pantanos de Villa, Lima - Perú. Ecol. Apl. 5 (1-2):119 - 125.

Vizcarra, J. K. (2008). Composición y conservación de las aves en los humedales de Ite, suroeste de Perú. Boletín Chileno de Ornitología, 14(2), 59-80. 